\title{
Estudo de utilização de medicamentos parenterais em uma unidade de internação pediátrica de um hospital universitário
}

\author{
Maria Clara Padovani de Souza ${ }^{* 1}$, Marta Aparecida Goulart ${ }^{1}$, Viviane Rosado ${ }^{2}$, \\ Adriano Max Moreira Reis ${ }^{3}$
}

${ }^{1}$ Unidade Funcional Farmácia, Hospital das Clínicas, Universidade Federal de Minas Gerais, ${ }^{2}$ Comissão de Controle de Infecção do Hospital das Clínicas da Universidade Federal de Minas Gerais, ${ }^{3}$ Departamento de Produtos

Farmacêuticos, Faculdade de Farmácia, Universidade Federal de Minas Gerais

*Correpondência:

M. C. P. Souza

Unidade Funcional Farmácia

Hospital das Clínicas

Universidade Federal de Minas Gerais

Av. Professor Alfredo Balena, 110 -

Santa Efigênia

30130-100 - Belo Horizonte - MG,

Brasil

Email: clarapadovani@ig.com.br
A administração de medicamentos parenterais tem grande importância na assistência pediátrica e no risco para aquisição de infecções hospitalares. Este estudo observacional transversal visou descrever a utilização de medicamentos em uma unidade de internação pediátrica. Elaborou-se um instrumento de coleta de dados, as variáveis relativas à farmacoterapia foram coletadas da prescrição médica e a análise estatística descritiva foi realizada no SPSS. A amostra foi constituída de 75 pacientes pediátricos, sendo $56,0 \%$ do sexo masculino; apresentando como predominante a faixa etária de lactentes. O tempo de internação mais freqüente foi maior que 20 dias $(24,0 \%)$ seguida de 6 a 10 dias $(21,3 \%)$ e menos de 3 dias (17,3\%). A via parenteral foi prescrita para 56 pacientes $(74,7 \%)$ e 19 (25,3\%) utilizaram outras vias ou não utilizaram medicamentos; sendo que a via parenteral endovenosa foi utilizada por 52 (92,9\%) dos pacientes. O número de medicamentos parenterais prescritos abrangeu 47 fármacos. A média do número de medicamentos por paciente foi quatro, o que implica em exigência de maior tempo da equipe de enfermagem em atividades relacionadas à administração de medicamentos. O número elevado de medicamentos prescritos desperta preocupações em relação à segurança. O farmacêutico deve estimular a conversão da via de administração parenteral para a oral.

\section{INTRODUÇÃO}

A administração de um medicamento pela via parenteral é um dos dispositivos eficazes no tratamento da saúde, mas quando a opção é a administração por via endovenosa é importante considerar o potencial significativo para o aumento do risco de doenças iatrogênicas, par- ticularmente bacteremias e candidemias (Crnich, Maki, 2002). Mais de 50,0\% de todas as bacteremias epidêmicas hospitalares ou candidemias relatadas na literatura, entre 1965 e 1991, foram derivadas de algum tipo de acesso vascular (Phyllips, 2000).

A utilização racional de medicamentos é pré-requisito para a prevenção e controle das infecções hospitalares 
(Milkovich, 2000; Guven, Uzun, 2003). É importante destacar que medicamentos parenterais são amplamente utilizados na prática clínica, porém contribuem para o aumento dos custos de internação e o uso inapropriado determina o aumento da ocorrência de eventos adversos (Castro, 2002).

As taxas de infecções apresentam variações significativas entre as diferentes populações pediátricas, principalmente devido à distribuição dos fatores de risco extrínseco e intrínseco para aquisição de infecções hospitalares (Jarvis, 2004).

Os principais fatores de risco intrínsecos para aquisição de infecção hospitalar são: prematuridade, idade, peso ao nascer, doenças congênitas e estado imunitário. Entre os fatores de risco extrínsecos destacam-se os procedimentos invasivos, incluindo, portanto, a terapia medicamentosa endovenosa (Jarvis, 2004).

A utilização de medicamentos em hospitais se inicia com a prescrição e envolve outras etapas diretamente relacionadas com os processos de trabalho da farmácia e da enfermagem. Entre essas etapas podemos destacar o armazenamento, a dispensação, o preparo e a administração dos medicamentos. Para as ações de controle de infecções hospitalares, a utilização de medicamentos parenterais é objeto de grande interesse. O conhecimento do processo de utilização de medicamentos contribui para definição de estratégias de melhoria do mesmo, com impacto significativo para a instituição de saúde, a qualidade do cuidado e o custo assistencial.

Empregando o referencial da farmacoepidemiologia, os estudos de utilização de medicamentos são investigações que permitem conhecer o seu perfil de utilização, produzindo conhecimentos que possibilitam intervenções que visam a promoção do uso racional de medicamentos, contribuindo para aprimorar a qualidade e a segurança da assistência (Osório-de-Castro, 2000; Carvalho et al., 2007).

Diante do exposto, delineou-se este estudo de utilização de medicamentos parenterais em uma unidade de internação pediátrica de um hospital universitário. O objetivo do estudo foi descrever a utilização de medicamentos parenterais, nessa unidade, sob a perspectiva da segurança de medicamentos e do controle de infecções hospitalares.

\section{MATERIAL E MÉTODOS}

Trata-se de um estudo observacional, descritivo, transversal, que analisou a utilização de medicamentos parenterais na unidade de internação pediátrica de um hospital universitário.

Realizou-se um estudo piloto prévio para testar o instrumento de coleta de dados e, em seguida, procederam- se adaptações no instrumento. A coleta de dados foi realizada no mês de setembro de 2005, durante três dias com intervalos de sete dias. O paciente que permaneceu internado, nos três dias de coleta, foi considerado um paciente, agrupando-se as informações dos três dias, para fins de análise. A amostragem foi não-probabilística, pois abrangia todas as prescrições de pacientes que faziam uso de farmacoterapia, no período investigado. O intervalo de coleta dos dados foi definido baseado na taxa de permanência calculada pelo Serviço de Arquivo Médico e Estatística do hospital, visando obter representatividade do tempo de internação na unidade estudada.

A partir do sistema de distribuição de medicamentos individualizado direto, adotado pela unidade de farmácia do hospital, foram identificados os pacientes que faziam uso de farmacoterapia. As variáveis relativas aos medicamentos parenterais prescritos foram coletadas através das segundas vias de prescrição. A classificação farmacológica foi realizada segundo o sistema anatômico terapêutico químico - ATC do "WHO Collaborating Centre for Drug Statistics Methodology", órgão da Organização Mundial da Saúde (WHO, 2006). Os medicamentos foram classificados segundo os níveis 1 (grupo anatômico principal) e 4 (subgrupo químico) da referida classificação. As variáveis relativas aos pacientes foram obtidas através do sistema informatizado de internação. Os pacientes não foram entrevistados e nem identificados. A classificação da faixa etária foi realizada segundo Leão (2005). O estudo foi autorizado pela instituição e aprovado pelo Comitê de Ética em Pesquisa da Universidade.

Os dados foram digitados em uma planilha de Excel versão 2000 e a análise estatística descritiva abrangendo a determinação de freqüência absoluta e relativa foi realizada empregando o software SPSS10.0.

\section{RESULTADOS}

A população amostral, considerando os três dias de coleta na unidade de internação pediátrica foi de 75 pacientes. Dos pacientes internados, $42(56,0 \%)$ eram do sexo masculino e $33(44,0 \%)$ do sexo feminino.

A idade das crianças incluídas no estudo variou de 14 dias a 16 anos, sendo a média de 5 anos e 8 meses e o desvio padrão de 4 anos e 9 meses. Os lactentes ( 1 mês até 23 meses) corresponderam a $35,1 \%$ dos pacientes, seguido das crianças (6 até 12 anos ) 31,1\% e dos pré-escolares (2 até 5 anos) 20,3\%. Os adolescentes (13 até 18 anos) representaram 10,8\% e os recém-nascidos (menos de 28 dias) representaram $2,7 \%$.

A faixa de tempo de internação encontrada com maior freqüência $(24,0 \%)$ foi dos pacientes hospitalizados 
por mais de 20 dias; seguido por período de 6 a 10 dias $(21,3 \%)$ e internação menor que 3 dias $(17,3 \%), 3$ a 5 dias $(16,0 \%)$ de 11 a 15 dias $(13,3 \%)$ e 16 a 20 dias $(8,0 \%)$.

A via parenteral foi empregada para administração de medicamentos para 56 pacientes $(74,7 \%)$ e $19(25,3 \%)$ utilizaram outras vias ou não estavam fazendo uso de medicamentos. A via endovenosa foi utilizada por 52 dos pacientes $(92,9 \%)$, a via subcutânea por 1 paciente $(1,8 \%)$ e o uso da via endovenosa e subcutânea ao mesmo tempo foi empregado em 3 pacientes $(5,4 \%)$.

A Tabela I apresenta a distribuição de número de medicamentos parenterais prescritos por pacientes. A média do número de medicamentos parenterais prescritos foi 4 , com desvio padrão de 2 , a amplitude foi de 1 a 9 por paciente, as maiores freqüências foram de $2(17,9 \%)$, $3(17,9 \%)$ e $5(16,1 \%)$ medicamentos.

TABELA I - Distribuição do número de medicamentos parenterais prescritos por pacientes, Belo Horizonte, 2005, $\mathrm{N}=56$

\begin{tabular}{lcc}
\hline $\begin{array}{l}\text { Número de medicamentos } \\
\text { prescritos por paciente }\end{array}$ & Freqüência & $\begin{array}{c}\text { Porcentagem } \\
(\%)\end{array}$ \\
\hline 1 & 9 & 16,1 \\
2 & 10 & 17,9 \\
3 & 10 & 17,9 \\
4 & 7 & 12,5 \\
5 & 9 & 16,1 \\
6 & 5 & 8,9 \\
7 & 1 & 1,8 \\
8 & 3 & 5,4 \\
9 & 2 & 3,6 \\
Total & 56 & 100,0 \\
\hline
\end{tabular}

O perfil de medicamentos parenterais prescritos abrangeu 47 fármacos. As Tabelas II e III apresentam a distribuição dos medicamentos parenterais prescritos para os pacientes, segundo a classificação anatômica, terapêutica, química, nível 1 e 4, respectivamente.

Os medicamentos antiinfecciosos parenterais foram prescritos para $34(60,7 \%)$ dos 56 pacientes que fizeram uso da via parenteral. Na Tabela IV, apresenta-se a distribuição do número de medicamentos antiinfecciosos parenterais prescritos por pacientes.

A duração de tratamento com antiinfecciosos mais freqüente foi de 11 a 15 dias $(39,0 \%)$, seguido de 6 a 10 dias $(27,1 \%)$, de 16 a 20 dias $(13,6 \%)$, inferior a 6 dias $(15,2 \%)$ e mais de 20 dias $(5,1 \%)$. O tempo de tratamento corresponde ao dia de início, até o dia da última data de coleta de dados. O início do tratamento foi identificado
TABELA II - Distribuição dos medicamentos parenterais prescritos segundo a classificação anatômica terapêutica química nível 1, Belo Horizonte, 2005; N = 224

\begin{tabular}{|c|c|c|}
\hline Classificação ATC & Freqüência & $\begin{array}{c}\text { Porcentagem } \\
(\%)\end{array}$ \\
\hline $\begin{array}{l}\text { A-Aparelho Digestivo e } \\
\text { Metabolismo }\end{array}$ & 44 & 19,6 \\
\hline $\begin{array}{l}\text { B - Sangue e Órgãos } \\
\text { Hematopoiéticos }\end{array}$ & 43 & 19,2 \\
\hline C - Aparelho Cardiovascular & 06 & 2,7 \\
\hline H - Terapia Hormonal & 09 & 4,0 \\
\hline J - Terapia Antiinfecciosa & 70 & 31,2 \\
\hline $\begin{array}{l}\text { L - Terapia Antineoplásica e } \\
\text { Agentes Imunomoduladores }\end{array}$ & 02 & 0,9 \\
\hline N - Sistema Nervoso & $\begin{array}{c}50 \\
224\end{array}$ & $\begin{array}{c}22,4 \\
100,0\end{array}$ \\
\hline
\end{tabular}

pelos registros da farmácia hospitalar realizados para o programa de auditoria de antimicrobianos.

A freqüência da apresentação dos medicamentos parenterais prescritos, no período estudado, foi a seguinte: $69(30,8 \%)$ frascos ampola, $111(49,6 \%)$ ampolas e 44 $(19,6 \%)$ em frascos parenterais de grande volume.

Identificou-se que $47(66,2 \%)$ pacientes possuíam acesso venoso, sendo $18,4 \%$ por via central, $79,6 \%$ por via periférica e $2,0 \%$ dos pacientes estavam utilizando as duas vias, concomitantemente.

A quimioterapia antineoplásica foi utilizada por apenas $8(14,3 \%)$ dos 56 pacientes e somente $1(1,8 \%)$ paciente estava submetido à terapia de nutrição parenteral.

\section{DISCUSSÃO}

A distribuição das internações não foi uniforme para os dois sexos, tendo o sexo masculino maior proporção em relação ao sexo feminino. Outros pesquisadores nacionais detectaram também maior freqüência de internação de pacientes do gênero masculino (Castro et al., 2004; Chang et al., 2003).

Os lactentes ( 1 mês até 23 meses) e os pré-escolares (2 até 5 anos), conjuntamente corresponderam a 56,4\% dos pacientes estudados. Estes resultados são compatíveis com estudos do Fundo das Nações Unidas para Infância (UNICEF), referentes à morbimortalidade, em crianças menores de cinco anos (Castro et al., 2004). É importante destacar que o lactente foi a faixa etária mais prevalente, portanto no planejamento das ações de controle de infecções, medidas direcionadas à assistência a esse grupo devem ser previstas. A taxa de infecção, no primeiro ano de vida, parece ser duas vezes superior à taxa nos grupos de 
idades subseqüentes, sofrendo diminuição após os dez primeiros anos (Martins, 2001).

TABELA III - Distribuição dos medicamentos parenterais prescritos segundo classificação ATC - subnível 4, Belo Horizonte, 2005; $\mathrm{N}=224$

\begin{tabular}{|c|c|c|}
\hline Classes Terapêuticas & Freqüência & $\begin{array}{l}\text { Porcentagem } \\
(\%)\end{array}$ \\
\hline Pirazolonas & 44 & 19,6 \\
\hline Soluções eletrolíticas & 39 & 17,4 \\
\hline Propulsivos & 16 & 7,1 \\
\hline Glicopeptídios & 14 & 6,3 \\
\hline Carbapenêmicos & 12 & 5,4 \\
\hline $\begin{array}{l}\text { Aminoglicosídios } \\
\text { (exceto estreptomicina) }\end{array}$ & 11 & 4,9 \\
\hline $\begin{array}{l}\text { Antagonistas do receptor } \\
\mathrm{H} 2 \text { da histamina }\end{array}$ & 9 & 4,0 \\
\hline Antagonistas da serotonina & 9 & 4,0 \\
\hline Glicocorticóides & 7 & 3,1 \\
\hline Cefalosporinas $3^{\mathrm{a}}$ Geração & 7 & 3,1 \\
\hline Derivados imidazólicos & 7 & 3,1 \\
\hline Alcalóides naturais do ópio & 5 & 2,2 \\
\hline Diuréticos de alça ascendente & 5 & 2,2 \\
\hline Inibidores da bomba de próton & 5 & 2,2 \\
\hline Antibiótico antifúngico & 4 & 1,8 \\
\hline Cefalosporinas $1^{\mathrm{a}}$ Geração & 4 & 1,8 \\
\hline $\begin{array}{l}\text { Penicilinas resistentes à } \\
\text { beta-lactamase }\end{array}$ & 4 & 1,8 \\
\hline $\begin{array}{l}\text { Insulina e análogos de } \\
\text { ação intermediária }\end{array}$ & 2 & 0,9 \\
\hline Insulina e análogos de ação rápida & 2 & 0,9 \\
\hline Outras preparações antianêmicas & 2 & 0,9 \\
\hline Penicilinas de amplo espectro & 2 & 0,9 \\
\hline Sulfametoxazol+Trimetropim & 2 & 0,9 \\
\hline Penicilinas sensíveis à beta-lactamase & 1 & 0,4 \\
\hline $\begin{array}{l}\text { Alcalóides da Beladona } \\
\text { semi-sintéticos, compostos }\end{array}$ & 1 & 0,4 \\
\hline Agentes dopaminérgios e adrenérgicos & os 1 & 0,4 \\
\hline Análogos de pirimidina & 1 & 0,4 \\
\hline Análogos de ácido fólico & 1 & 0,4 \\
\hline Barbiturato e derivados & 1 & 0,4 \\
\hline Cefalosporinas $4^{\mathrm{a}}$ Geração & 1 & 0,4 \\
\hline Fluoroquinolonas & 1 & 0,4 \\
\hline Lincosamidas & 1 & 0,4 \\
\hline Vasopressinas e análogos & 1 & 0,4 \\
\hline Vitamina K & 1 & 0,4 \\
\hline $\begin{array}{l}\text { Preparações parenterais } \\
\text { de ferro trivalente }\end{array}$ & 1 & 0,4 \\
\hline Total & 224 & 100,0 \\
\hline
\end{tabular}

TABELA IV - Distribuição do número de medicamentos antiinfecciosos, parenterais, prescritos por paciente, Belo Horizonte, 2005; $\mathrm{N}=56$.

\begin{tabular}{lcc}
\hline $\begin{array}{l}\text { Número de antiinfecciosos } \\
\text { parenterais prescritos }\end{array}$ & Freqüência & $\begin{array}{c}\text { Porcentagem } \\
(\%)\end{array}$ \\
\hline Nenhum & 22,0 & 39,3 \\
1 & 13,0 & 23,2 \\
2 & 14,0 & 25,0 \\
3 & 7,0 & 12,5 \\
Total & 56,0 & 100,0 \\
\hline
\end{tabular}

O risco das crianças adquirirem infecção incluem: fatores relativos ao próprio paciente (imaturidade do estado imunológico, condição nutricional, risco maior de infecções no primeiro ano de vida) e fatores relativos à conduta no período de hospitalização como número de procedimentos invasivos e período prolongado de internação (Elward, Mcgann, 2002).

A utilização de via parenteral por $74,7 \%$ dos pacientes pode ser atribuída às características dos pacientes atendidos no hospital (universitário, de nível terciário), em sua maioria portadores de doenças crônico-degenerativas e de alta complexidade. Outro fator a ser considerado, advém do tipo de população estudada, a pediátrica, que por características específicas, requer maior administração de medicamentos por via endovenosa, comparativamente ao adulto, do que por outras vias mais comumente utilizadas como a intramuscular e oral(Peterline et al., 2003).

A ausência de administração de medicamentos por via intramuscular, no período estudado, é explicável pelo fato de que a massa muscular da criança é, aproximadamente, $38,0 \%$ menor que a do adulto, e a composição corporal muscular do recém-nascido é de, aproximadamente, $25,0 \%$, enquanto que no adulto, varia em torno de $40,0 \%$. Devido à pequena massa muscular, poucos sítios são recomendados para as injeções intramusculares. O fluxo sangüíneo muscular irregular, também pode afetar a absorção de determinados fármacos em crianças (Peterlini et al., 2003). Esses fatores em conjunto minimizam a indicação de medicamentos, por via intramuscular, em pediatria.

O número médio de quatro medicamentos parenterais por paciente, exige da equipe de enfermagem da unidade de internação pediátrica dedicação de parte significativa do tempo de trabalho aos pacientes em terapia endovenosa. Esse número elevado de medicamentos prescritos despertam preocupações também em relação à segurança, pois é um fator significativo para o desenvolvimento de reações adversas a medicamentos (Santos, Coelho, 2005). 
O perfil etário destaca a relevância de ações de controle de infecções em pediatria, pois vários estudos demonstram que a freqüência de infecções é inversamente proporcional à idade dos pacientes (Jarvis, 1987; Chang et al., 2003).

O tempo de internação prolongado é um fator que favorece a ocorrência de infecções hospitalares (Jarvis, 1987; Moraes et al., 2000). Os resultados demonstram que o tempo de internação da população estudada é longo, considerando que a maioria dos pacientes permaneceu hospitalizada por mais de 6 dias, sendo que para $24 \%$ dos pacientes, o período de internação foi superior a 20 dias,. Esse resultado é preocupante diante da maior susceptibilidade dos pacientes pediátricos ao risco de infecções hospitalares. Quanto maior o período de internação, maior exposição a procedimentos invasivos e à flora bacteriana hospitalar, o que pode levar ao aparecimento de bactérias multirresistentes. O tempo médio de permanência até o aparecimento de uma infecção varia entre a segunda e terceira semana de internação, podendo ser reduzido há poucos dias até uma semana, quando ocorrem surtos de infecções virais respiratórias ou gastrintestinais (Jarvis, 1987; Martins, 2001).

O perfil de medicamentos utilizados pelos pacientes classificados segundo critério ATC nível 1, é bastante similar aos resultados de estudo realizado em um hospital pediátrico espanhol. No referido estudo, os grupos mais utilizados foram J, N, C e A em ordem decrescente (Danés et al., 2002). De acordo com os resultados apresentados na Tabela II, foi observado que no arsenal terapêutico empregado na Unidade de Internação Pediátrica os antiinfecciosos (Grupo J) corresponderam à classe terapêutica de medicamentos parenterais mais prescritos.

Um estudo farmacoepidemiológico publicado por Santos e Coelho (2005) detectou que os antiinfecciosos para uso sistêmico foi a classe terapêutica mais prescrita em hospital infantil do Ceará. Fica evidente a relevância desta classe para a assistência ao paciente pediátrico e a necessidade de implementar ações para a promoção do uso racional desses medicamentos. A Comissão de Controle de Infecção do hospital estudado já adota uma política de uso racional de antimicrobianos, que desenvolve entre outras ações, a auditoria e treinamentos de profissionais.

A análise dos medicamentos, segundo o nível ATC 4 (Tabela III), demonstra que a dipirona, fármaco do grupo das pirazolonas, foi o medicamento parenteral mais prescrito. Vale ressaltar que, no âmbito hospitalar, esse fármaco é prescrito como sintomático, não implicando necessariamente em administração. O mesmo ocorre com a metoclopramida, classificada pela ATC como propulsivos. As classes de fármacos antimicrobianos empregados na unidade de internação pediátrica ficam melhor elucidadas com a análise pelo nível 4 da ATC.
Os antibacterianos mais utilizados na Unidade de Internação Pediátrica foram glicopeptídios, carbapenêmicos, cefalosporinas de terceira geração e aminoglicosídios, cujo espectro de ação é adequado para as infecções mais prevalentes em crianças hospitalizadas. Segundo a literatura, a maioria dos relatos indica que os Staphylococcus e os bastonetes gram negativos são responsáveis por maior parte das infecções nosocomiais em crianças, microrganismos para os quais os agentes terapêuticos encontrados são úteis (Martins, 2001).

Os antifúngicos foi a segunda categoria de agentes infecciosos mais utilizados na unidade de internação pediátrica. A incidência das infecções fúngicas está aumentando, significativamente, nos últimos anos. Paralelamente, observa-se maior gravidade dessas infecções, inclusive em crianças, principalmente nos pacientes hospitalizados. Alguns fatores justificam as alterações na epidemiologia das infecções fúngicas, tais como: avanços nos procedimentos cirúrgicos e no tratamento das neoplasias; o uso freqüente de procedimentos invasivos e a melhoria da sobrevida dos grupos de riscos para infecções fúngicas; pacientes com diabetes mellitus; o aumento de pacientes onco-hematológicos e receptores de transplantes (Martins, 2001). Entre os fatores citados, vários estão presentes no perfil assistencial da unidade de internação pediátrica estudada e justificam a utilização de antifúngicos.

No estudo, foi identificado que os medicamentos empregados apresentavam-se em maior proporção nas formas farmacêuticas de uso parenteral e as sólidas de uso oral. A administração parenteral de medicamentos apresenta potencial significativo para o aumento do risco de doenças iatrogênicas, particularmente bacteremias e candidemias (Crnich, Maki, 2002). Diante da relevância clínica do emprego das formas parenterais, evidenciada em diversos estudos, é essencial a administração adequada desses medicamentos. Uma ferramenta que contribui para alcançar esse objetivo e reduzir os riscos citados é a adoção das boas práticas de terapia endovenosa em serviços de saúde preconizadas pela Agência Nacional de Vigilância Sanitária -ANVISA(Brasil, 2003). Na unidade de internação pediátrica do hospital investigado, não foi identificada a disponibilidade de procedimentos escritos para orientar a administração de medicamentos parenterais, como preconiza as boas práticas da ANVISA. Porém, na unidade de internação pediátrica, existe um manual com orientações sobre reconstituição, diluição, incompatibilidades, estabilidade físico-química e microbiológica de medicamentos parenterais. A elaboração do manual foi realizada por farmacêuticos em parceria com enfermeiros e abrange um número significativo de medicamentos, sendo instrumento adequado para orientar o preparo e administração de medicamentos parenterais. 
O farmacêutico, dentro da perspectiva do trabalho multidisciplinar, deve assessorar a equipe de enfermagem em aspectos relacionados à diluição, incompatibilidades $\mathrm{e}$ interações. O manual de medicamentos parenterais desenvolvido na instituição estudada é um exemplo de trabalho multidisciplinar, com impacto nas ações de controle de infecções e qualidade da assistência.

$\mathrm{Na}$ escolha da via de administração de medicamentos, deve-se considerar a adequação ao estado clínico do paciente e as características farmacocinéticas do medicamento (Martin et al., 2000). A administração do medicamento, por via oral, depende de existir apresentação pediátrica e da possibilidade de absorção pelo trato gastrintestinal, que pode estar prejudicada e do quadro clínico, em que a utilização da via oral se torna impossível (Peterlini et al., 2003). Havendo inviabilidade de utilização da via oral, pode-se recorrer à administração do medicamento por via parenteral.

Quanto à administração de medicamentos por via oral em crianças, sabe-se que as menores de seis anos, principalmente, não utilizam cápsulas ou comprimidos, devido à dificuldade de deglutição. Alguns desses medicamentos são triturados ou rediluídos para administração, porém, sem a realização de estudos que indiquem a validade e qualidade desses procedimentos, o que pode corroborar para o aumento da necessidade de se administrar medicamentos por via intravenosa em crianças (Nahata, 1999; Costa et al., 2005).

No mercado farmacêutico, há os medicamentos parenterais que, também, não são adequados à faixa etária pediátrica. Estudo descritivo realizado em hospital pediátrico de São Paulo identificou que a falta de apresentação pediátrica gerou o aumento dos custos de atendimento. Nenhum dos 41 medicamentos prescritos possuía apresentação pediátrica, acarretando em alguns casos, maior manipulação durante o preparo, risco de contaminação e perda de estabilidade (Peterlini et al., 2003).

Considera-se que a falta de formulações e apresentações de medicamentos especificamente direcionados à faixa etária pediátrica, além de preocupante, constitui um desafio na busca da qualidade de assistência prestada à criança. As equipes de enfermagem, de farmacêuticos, de médicos e de gestores dos serviços de saúde devem direcionar esforços para modificar esse cenário.

Em alguns hospitais, uma estratégia criada para diminuir os impactos desse problema refere-se à implantação de centrais de preparo de misturas endovenosas. A centralização do preparo promove utilização mais racional de medicamentos nos hospitais.

O tempo do tratamento antimicrobiano dos pacientes internados na unidade de internação pediátrica é prolonga- do. Uma estratégia para incentivar a redução do tempo de tratamento com medicamentos parenterais, em especial com antimicrobianos, é a terapia seqüencial.

Terapia seqüencial é o termo empregado para designar a conversão do tratamento antimicrobiano por via endovenosa para via oral, empregando o mesmo fármaco. Se a conversão ocorrer empregando outro fármaco da mesma classe farmacológica denomina-se terapia de transferência (switch therapy). Freqüentemente, os termos são empregados de forma intercambiável porque são baseados no princípio de que a via oral como alternativa terapêutica tem eficácia comparável à via parenteral (Lelekis, Gould, 2001).

A diminuição das complicações associadas à terapia endovenosa, o conforto da administração por via oral e a possibilidade de alta hospitalar precoce também são fatores que mostram a importância da terapia seqüencial para o controle de infecções hospitalares e gestão dos custos assistenciais ( Lelekis, Gould, 2001).

Um número significativo de antimicrobianos de uso pediátrico possuem formas farmacêuticas para uso oral e parenteral facilitando a terapia seqüencial. Estes antimicrobianos incluem, penicilinas, com ou sem inibidores da beta-lactamase, macrolídeos, imidazólicos, cefalosporinas, sulfonamidas, trimetroprim e outros.

A implementação de programa de terapia seqüencial envolve a comissão de controle de infecção hospitalar, a comissão de farmácia e terapêutica e o serviço de farmácia. Para o êxito do programa recomenda-se que haja a definição prévia dos critérios e condições clínicas para conversão para via oral, baseadas em sólidas evidências científicas (Milkovich, 2000; Lelekis, Gould; 2001; Foz, 2003).

$\mathrm{Na}$ assistência à criança hospitalizada, o emprego da via parenteral é indicado em função do diagnóstico e da estratégia terapêutica a ser empregada. Porém, é importante destacar que com a evolução do quadro clínico, a terapia seqüencial deve ser viabilizada, pois contribui para a alta precoce, diminuindo o tempo de permanência e os riscos de infecção hospitalar. Assim, os custos para o sistema de saúde são reduzidos e a criança e sua família são beneficiadas nos aspectos emocionais e sociais.

A quimioterapia antineoplásica e a nutrição parenteral são prescritas em formulário especial no hospital e durante a coleta de dados foram identificados os pacientes em uso dessas terapias, ou seja, através dos registros da farmácia. O risco de contaminação existe durante o preparo se a técnica asséptica não é seguida e, também, durante a administração, pois microrganismos podem acessar os frascos pela entrada de ar, nos pontos de inserção do dispositivo endovenoso, pelo equipo ou na junção entre a via de administração e a conexão do cateter (Philips, 2001). No 
entanto, o risco de contaminações das soluções durante o preparo é reduzido, pois ambas são preparadas de forma centralizada em setor de preparações estéreis da farmácia do hospital, empregando-se tecnologia de salas limpas e cabine de fluxo laminar.

Na utilização da terapia de nutrição parenteral devese tomar o cuidado de observar o prazo de validade da formulação e conservá-la sob refrigeração. A presença de controle microbiológico das formulações e de programa de garantia de qualidade como já existe implementado no hospital estudado, é essencial para reduzir os riscos de contaminação.

A complexidade do cuidado prestado na instituição aliado às particularidades do paciente pediátrico podem explicar a alta freqüência de utilização de medicamentos parenterais. Esses fatores determinam também a porcentagem elevada de pacientes que fazem uso de antimicrobianos, mas, ainda é importante destacar que a avaliação prévia do auditor infectologista pediátrico é instrumento que contribui para o seu uso mais adequado. Estudos de utilização de medicamento do tipo prescrição - indicação podem ser desenvolvidos para elucidar a racionalidade da utilização desta classe farmacológica na instituição investigada.

\section{CONCLUSÕES}

Em pediatria, a administração parenteral de medicamentos é ferramenta importante no processo de assistência e torna o paciente mais propenso às infecções hospitalares se não for utilizada respeitando-se as boas práticas de terapia parenteral.

A presença dos medicamentos do grupo J, antiinfecciosos, entre as classes ATC mais utilizadas, é explicada pelo perfil assistencial da unidade de internação pediátrica que inclui pacientes submetidos a procedimentos invasivos, oncohematológicos e portadores de doenças crônicas graves.

O estudo permitiu conhecer a utilização de medicamentos parenterais na unidade de internação pediátrica, evidenciando aspectos para a melhoria da qualidade assistencial, como a implementação da terapia seqüencial. Uma maior integração da equipe de saúde contribui para melhorar o nível da qualidade da utilização dos medicamentos em pediatria.

\section{ABSTRACT}

\section{Study utilization of parenteral medications in pediatric unit of universitary hospital}

Parenteral drug administration has clinical relevance in pediatric care and the risk of acquire nosocomial infection. The purpose of this observational crosssectional study was to describe the utilization of parenteral drugs in a pediatric unit. A research questionnaire was created. The variables about pharmacotherapy were collect in prescriptions. The descriptive statistical analysis was performed using SPSS A total of 75 patients were admitted, $56 \%$ were male. The age range most frequently was the infants. The length of hospitalization was over 20 days (24.0\%), 6 to 10 days $(21.3 \%)$ and less than 3 days (17.3\%). Parenteral route was used to $56(74.7 \%)$ patients, so $19(25.3 \%)$ used other routes or did not use medicine. Intravenous route was used to $52(92.9 \%)$ patients. Overall, 47 different parenteral drugs were prescribed. The average of 4 drugs per patient requires more time of nursing team in activities related with drug administration. The high number of drug prescribed per patient raise concern about safety. The pharmacist should stimulate conversion from parenteral to oral administration .

UNITERMS: Pediatrics/assistance. Pharmacoepidemiology. Parenteral drugs/use. Pharmacotherapy. Hospital Infection.

\section{REFERÊNCIAS BIBLIOGRÁFICAS}

BRASIL. Agência Nacional de Vigilância Sanitária. Resolução RDC 45, de 12 de março de 2003. Aprova o regulamento técnico de boas práticas de utilização de soluções parenterais em serviços de saúde. Diário Oficial da União, Brasília, 13 mar. 2003. Seção 1, p.12.

CASTRO, M. S.; PILGER, D.; FERREIRA, M. B. C.; KOPITTKE, L.; Tendências na utilização de antimicrobianos em um hospital universitário, 1990-1996. Revista de Saúde Pública, São Paulo, v.36, n.5, p.553558, 2002.

CASTRO, L. L.; OSHIRO, M. L.; VICENTE, M. G.; CYMROT, R. Revisão retrospectiva de prontuários como estratégia de farmacovigilância. Saúde em Revista, v.6, n.12, p.37-46, 2004.

CARVALHO, J.M.; MAGARINOS-TORRES, R.; OSÓRIO-DE-CASTRO, C.G.S. Estudo de utilização de medicamentos em hospitais brasileiros: uma revisão bibliográfica. Rev.Bras. Farm., v.88, n.22, p.77-82, 2007.

CRNICH, C.J, MAKI, D.G. The promise of novel technology for the prevention of intravascular device-related bloodstream infection.I. Pathogenesis and short-term devices. Clin Infect Dis., v34, n.9, p.1232-1242, 2002. 
CHANG, M. R.; CARVALHO, N. C. P.; OLIVEIRA, A. L. L.; MONCADA, P.M.F. Surveillance of pediatric infections in a teaching hospital in Mato Grosso do sul, Brazil. Brazil J. Infect. Dis., v.7, n.20, p.149-160, 2003.

COSTA, P. Q.; LIMA, J. E. S.; COELHO, H. L. L. Medicamentos não padronizados para uso em crianças: problemática da utilização em hospital pediátrico do SUS em Fortaleza-CE. In: CONGRESSO BRASILEIRO SOBRE USO RACIONAL DE MEDICAMENTOS, 1 ., 2005, Porto Alegre. Livro de resumos. Porto Alegre: ANVISA/OPAS, 2005. p.74.

DANÉS, I.; VALLANO, A.; DE LA CRUZ, G.; JUÁREZ ,J. C.; ARNAU, J. M. Utilización de medicamentos y condiciones de uso recomendadas en pediatría. An. Esp. Pediatria, v.57, n.5, p.9414-9419, 2002.

MORAES, B. A.; CRAVO, C. A. N.; LOUREIRO, M.; SOLARI, C.A.;ASENSI, M. D. Análise epidemiológica de cepas bacterianas envolvidas em infecção hospitalar em um Hospital Universitário no Brasil. Rev. Inst. Med. Trop., v.42, n.4, p.201-207, 2000.

ELWARD, M. A.; MCGANN, K. A. Pediatric Infection: Steps to Reduce Nosocomial Infections in Children. Infect. Med., v.19, n.9, p.414-424, 2002.

FOZ E. Pharmacy - administered IV to oral therapeutic interchange program: development, implementation and cost - assessment. Hosp. Pharm., v.38, n.5, p.444-452, 2003.

GUVEN, G. S.; UZUN, O. Principles of good use of antibiotics in hospitals. J. Infect. Control., v.53, p.91-96, 2003.

JARVIS, W. R. Epidemiology of nosocomial infections in pediatric patients. Pediatr. Infec. Dis. J., v.6, n.4, p.34451,1987

JARVIS, W. R. Controlling healtcare-associated infections: the role of infection control and antimicrobial use practices. Semin. Pediatr. Infect. Dis., v.15, n.1, 2004.

LEÃO, E. Pediatria ambulatorial. 4. ed. Belo Horizonte: COOPMED, 2005. 1034 p.

LELEKIS, M.; GOULD, I. M. Sequential antibiotic therapy for cost containment in the hospital setting: why not? $J$. Infec. Control., v.48, p.249-257, 2001.
MARTIN, F. F.; GONZALEZ MARTINEZ, J. C.; DOMINGUEZ ULIBARRI, R. Estudio económico de la infección nosocomial en una unidad de cuidados intensivos pediátricos. Rev. Cubana de Pediatr., v.72, n.1, p.21-26, 2000.

MARTINS, M. A. Infecções no paciente pediátrico e no adolescente. In: Manual de infecção hospitalar: epidemiologia, prevenção e controle. 2 ed. Rio de Janeiro: Medsi, 2001. Cap.22, p.237 - 261.

MILKOVICH, G. The role of hospital pharmacist in cost control and antibiotic. Intern. J. Antimicrobial Agent., v.16, n.3, p.291-294, 2000.

NAHATA, M. C. Lack of pediatric drug formulations. Pediatrics., v.104, n.3, p.607-609, 1999.

OSÓRIO-DE-CASTRO, C. G. S. Estudos de utilização de medicamentos - noções básicas. Rio de Janeiro: Editora Fiocruz, 2000.92p.

PETERLINI, M. A. S.; CHAUD, M. N.; PEDREIRA, M. L. G. Órfãos de terapia medicamentosa: a administração de medicamentos por via intravenosa em crianças hospitalizadas. Rev. Latino-Am. Enfermagem, v.11, n.1, p.88-95, 2003.

PHILLIPS, L. D. Manual de Terapia Intravenosa. 2.ed. Porto Alegre: Artmed Editora, 2001. 551p.

SANTOS, D. B.; COELHO, H. L. L. Análise de prescrição de medicamentos em crianças hospitalizadas em Fortaleza. In: I CONGRESSO BRASILEIRO SOBRE USO RACIONAL DE MEDICAMENTOS, I, 2005, Porto Alegre. Livro de resumos. Porto Alegre: ANVISA/ OPAS, 2005. p.74.

WORLD HEALTH ORGANIZATION Collaborating Centre for Drug Statistics Methodology. Anatomical Therapeutic Chemical (ATC) index with Defined Daily Doses (DDDs). Disponível em: $<$ http://www.whocc.no/atcddd $>$. Acesso em: 10 mar. 2006

Recebido para publicação em 08 de outubro de 2007 Aceito para publicação em 30 de setembro de 2008 